\title{
GLOBAL BEHAVIOR OF A THIRD ORDER DIFFERENCE EQUATION
}

\author{
R. ABO-ZEID AND M. A. AL-SHABI
}

Abstract. The aim of this paper is to investigate the global stability and periodic nature of the positive solutions of the difference equation

$$
x_{n+1}=\frac{A+B x_{n-1}}{C+D x_{n} x_{n-2}}, \quad n=0,1,2, \ldots
$$

where $A, B$ are nonnegative real numbers and $C, D>0$.

\section{Introduction and preliminaries}

Difference equations, although their forms look very simple, it is extremely difficult to understand thoroughly the global behaviors of their solutions. One can refer to [1, 7]. The study of nonlinear rational difference equations of higher order is of paramount importance, since we still know so little about such equations. It is worthwhile to point out that although several approaches have been developed for finding the global character of difference equations $[7,8,9,10]$, relatively a large number of difference equations have not been thoroughly understood yet $[4,11,12]$.

Cinar [3] examined the global asymptotic stability of all positive solutions of the rational difference equation

$$
x_{n+1}=\frac{a x_{n-1}}{1+b x_{n} x_{n-1}}, \quad n=0,1,2, \ldots
$$

Xiaofan yang et all [13] investigated the global attractivity of all solutions of the difference equation

$$
x_{n+1}=\frac{a x_{n}+b x_{n-1}}{c+d x_{n} x_{n-1}}, \quad n=0,1,2, \ldots
$$

where $a \geq o, b, c, d>0$.

C. H. Gibbons et al. [6] investigated the global asymptotic behavior of the difference equation

$$
x_{n+1}=\frac{\alpha+\beta x_{n-1}}{\gamma+x_{n}}, \quad n=0,1,2, \ldots
$$

Corresponding author: R. Abo-Zeid.

2010 Mathematics Subject Classification. 39A11.

Key words and phrases. Difference equation, periodic solution, globally asymptotically stable. 
where $\beta>0$ and $\alpha, \gamma \geq 0$.

In [2] we have discussed the global asymptotic stability of the difference equation

$$
x_{n+1}=\frac{A x_{n-2 r-1}}{B+C x_{n-2 l} x_{n-2 k}}, \quad n=0,1,2, \ldots
$$

where $A, B, C$ are nonnegative real numbers and $r, l, k$ are nonnegative integers such that $l \leq k$ and $r \leq k$.

In this paper, we study the global asymptotic stability of all solutions of the difference equation

$$
x_{n+1}=\frac{A+B x_{n-1}}{C+D x_{n} x_{n-2}}, \quad n=0,1,2, \ldots
$$

where where $A, B$ are nonnegative real numbers and $C, D>0$.

We give some preliminaries which will be needed in this paper.

Consider the difference equation

$$
x_{n+1}=f\left(x_{n}, x_{n-1}, \ldots, x_{n-k}\right), \quad n=0,1, \ldots
$$

where $f: R^{k+1} \rightarrow R$.

Definition 1.1 ([7]). An equilibrium point for equation (1.3) is a point $\bar{x} \in R$ such that $\bar{x}=$ $f(\bar{x}, \bar{x}, \ldots, \bar{x})$.

Definition 1.2 ([7]). (1) An equilibrium point $\bar{x}$ for equation (1.3) is called locally stable if for every $\epsilon>0$, there exists a $\delta>0$ such that every solution $\left\{x_{n}\right\}$ with initial conditions $\left.x_{-k}, x_{-k+1}, \ldots, x_{0} \in\right] \bar{x}-\delta, \bar{x}+\delta$ [is such that $\left.x_{n} \in\right] \bar{x}-\epsilon, \bar{x}+\epsilon[$ for all $n \in \mathbb{N}$. Otherwise $\bar{x}$ is said to be unstable.

(2) The equilibrium point $\bar{x}$ of equation (1.3) is called locally asymptotically stable if it is locally stable and there exists $\gamma>0$ such that for any initial conditions $x_{-k}, x_{-k+1}, \ldots, x_{0} \in$ ] $\bar{x}-\gamma, \bar{x}+\gamma$ [, the corresponding solution $\left\{x_{n}\right\}$ tends to $\bar{x}$.

(3) An equilibrium point $\bar{x}$ for equation (1.3) is called a global attractor if every solution $\left\{x_{n}\right\}$ converges to $\bar{x}$ as $n \rightarrow \infty$.

(4) The equilibrium point $\bar{x}$ for equation (1.3) is called globally asymptotically stable if it is locally asymptotically stable and global attractor.

Suppose that $f$ is continuously differentiable in some open neighborhood of $\bar{x}$. Let

$$
a_{i}=\frac{\partial f}{\partial x_{n-i}}(\bar{x}, \ldots, \bar{x}), \quad \text { for } \quad i=0,1, \ldots, k
$$


denote the partial derivatives of $f\left(x_{n}, x_{n-1}, \ldots, x_{n-k}\right)$ with respect to $x_{n-i}$ evaluated at the equilibrium point $\bar{x}$ of equation (1.3). Then the equation

$$
z_{n+1}=\sum_{i=0}^{k} a_{i} z_{n-i}, \quad n=0,1, \ldots
$$

is called the linearized equation associated with equation (1.3) about the equilibrium point $\bar{x}$, and the equation

$$
\lambda^{k+1}-\sum_{i=0}^{k} a_{i} \lambda^{k-i}=0
$$

is called the characteristic equation associated with equation (1.4) about the equilibrium point $\bar{x}$.

Theorem 1.3 ([7]). Assume that $f$ is a $C^{1}$ function and let $\bar{x}$ be an equilibrium point of equation (1.3). Then the following statements are true:

(1) If all roots of equation (1.5) lie in the open disk $|\lambda|<1$, then $\bar{x}$ is locally asymptotically stable.

(2) If at least one root of equation (1.5) has absolute value greater than one, then $\bar{x}$ is unstable.

Theorem $1.4([5])$. Assume that $\sum_{i=0}^{k}\left|a_{i}\right|<1$. Then every root of equation (1.5) has absolute value less than one.

Definition 1.5 ([11]). A positive semicycle of a solution $\left\{x_{n}\right\}_{n=-k}^{\infty}$ of equation (1.3) consists of a "string" of terms $\left\{x_{l}, x_{l+1}, \ldots, x_{m}\right\}$, all greater than or equal to the equilibrium $\bar{x}$, with $l \geq-1$ and $m \leq \infty$ and such that

$$
\text { either } l=-k, \quad \text { or } l>-k \text { and } x_{l-1}<\bar{x}
$$

and

$$
\text { either } m=\infty \text {, or } m<\infty \text { and } x_{m+1}<\bar{x} \text {. }
$$

Definition 1.6 ([11]). A negative semicycle of a solution $\left\{x_{n}\right\}_{n=-k}^{\infty}$ of equation (1.3) consists of a "string" of terms $\left\{x_{l}, x_{l+1}, \ldots, x_{m}\right\}$, all less than or equal to the equilibrium $\bar{x}$, with $l \geq-1$ and $m \leq \infty$ and such that

$$
\text { either } l=-k, \quad \text { or } l>-k \text { and } x_{l-1} \geq \bar{x}
$$

and

$$
\text { either } m=\infty \text {, or } m<\infty \text { and } x_{m+1} \geq \bar{x} \text {. }
$$

The change of variables $x_{n}=\sqrt{\frac{C}{D}} y_{n}$ reduces equation (1.2) to the difference equation

$$
y_{n+1}=\frac{\alpha+\beta y_{n-1}}{1+y_{n} y_{n-2}}, \quad n=0,1,2, \ldots
$$


where $\alpha=\frac{A}{C} \sqrt{\frac{D}{C}}, \beta=\frac{B}{C}$.

When $\alpha=0$, equation (1.6) becomes

$$
y_{n+1}=\frac{\beta y_{n-1}}{1+y_{n} y_{n-2}}, \quad n=0,1,2, \ldots
$$

This equation is a special case of (1.1) with $r=0, l=0$ and $k=1$.

\section{Linearized stability analysis}

Now we determine the equilibrium points of equation (1.6) and discuss their local asymptotic behavior. It is clear that the values of the equilibrium points depends on $\alpha$ and $\beta$.

Lemma 2.1. Assume that the function $f(x)=x^{3}+(1-\beta) x-\alpha$, where $\alpha$ and $\beta$ are positive real numbers. Then the following statements are true:

(1) If $\beta<1$, then $f(x)$ has a unique zero $x^{*}>0$,

(2) If $\beta>1$, then we have the following:

- If $\alpha>2\left(\frac{\beta-1}{3}\right)^{\frac{3}{2}}$, then there exists a unique zero $x^{*}>0$.

- If $\alpha<2\left(\frac{\beta-1}{3}\right)^{\frac{3}{2}}$, then there exist three zeros $x^{*}>\sqrt{\beta-1},-\sqrt{\frac{\beta-1}{3}}<x^{* *}<0$ and $-\sqrt{\beta-1}<$ $x^{* * *}<-\sqrt{\frac{\beta-1}{3}}$

Proof. We can see that the function $f(x)$ has a positive zero whatever the value of $\beta$. As $f^{\prime}(x)=3 x^{2}+1-\beta$, the function $f(x)$ is increasing everywhere when $\beta<1$. Therefore, the function $f(x)$ has a unique (positive) zero.

When $\beta>1$, the zeros of the function $f(x)$ depends on the relation between $\alpha$ and $\beta$.

Clear that, the equilibrium points of equation (1.6) are the zeros of the function $f(\bar{y})=$ $\bar{y}^{3}+(1-\beta) \bar{y}-\alpha$. When $\beta<1$, equation (1.6) has a unique (positive) equilibrium point $\bar{y}$ such that $\bar{y}>\sqrt{1-\beta}$ if $\alpha>2(1-\beta)^{\frac{3}{2}}$ and $0<\bar{y}<\sqrt{1-\beta}$ if $\alpha<2(1-\beta)^{\frac{3}{2}}$. When $\beta>1$, equation (1.6) has a unique (positive) equilibrium point $\bar{y}>\sqrt{\beta-1}$ if $\alpha>2\left(\frac{\beta-1}{3}\right)^{\frac{3}{2}}$ and three equilibrium points $\bar{y}>\sqrt{\beta-1},-\sqrt{\frac{\beta-1}{3}}<\overline{y_{1}}<0$ and $-\sqrt{\beta-1}<\overline{y_{2}}<-\sqrt{\frac{\beta-1}{3}}$ if $\alpha<2\left(\frac{\beta-1}{3}\right)^{\frac{3}{2}}$.

We fucus our interest on the positive equilibrium point $\bar{y}$.

The linearized equation associated with equation (1.6) about $\bar{y}$ is

$$
z_{n+1}+\frac{\bar{y}^{2}}{1+\bar{y}^{2}} z_{n}-\frac{\beta}{1+\bar{y}^{2}} z_{n-1}+\frac{\bar{y}^{2}}{1+\bar{y}^{2}} z_{n-2}=0, \quad n=0,1,2, \ldots
$$

the characteristic equation associated with this equation is

$$
\lambda^{3}+\frac{\bar{y}^{2}}{1+\bar{y}^{2}} \lambda^{2}-\frac{\beta}{1+\bar{y}^{2}} \lambda+\frac{\bar{y}^{2}}{1+\bar{y}^{2}}=0 .
$$

We summarize the results of this section in the following theorem. 
Theorem 2.2. The following statements are true:

(1) If $\beta<1$, then

(a) $\bar{y}$ is locally asymptotically stable if $\alpha<2(1-\beta)^{\frac{3}{2}}$.

(b) $\bar{y}$ is a saddle point if $\alpha>2(1-\beta)^{\frac{3}{2}}$.

(2) If $\beta>1$, then $\bar{y}$ is is a saddle point.

\section{Proof.}

(1) Assume that $\beta<1$.

(a) If $\alpha<2(1-\beta)^{\frac{3}{2}}$, that is $\bar{y}<\sqrt{1-\beta}$, then using theorem (1.4) we get the result.

(b) Assume that $\alpha<2(1-\beta)^{\frac{3}{2}}$ and consider the function $g(\lambda)=\lambda^{3}+\frac{\bar{y}^{2}}{1+\bar{y}^{2}} \lambda^{2}-\frac{\beta}{1+\bar{y}^{2}} \lambda+\frac{\bar{y}^{2}}{1+\bar{y}^{2}}$. Clear that $g(\lambda)$ has a real root $\lambda_{1}$ in $(-\infty,-1)$. As $g\left(-\frac{\bar{y}^{2}}{1+\bar{y}^{2}}\right)>0$, we have $\lambda_{1}<-1<$ $-\frac{\bar{y}^{2}}{1+\bar{y}^{2}}$. That is $\left|\lambda_{1}\right|>\frac{\bar{y}^{2}}{1+\bar{y}^{2}}$.

But

$$
\frac{\bar{y}^{2}}{1+\bar{y}^{2}}=\left|\prod_{i=0}^{2} \lambda_{i}\right|=\left|\lambda_{1}\right|\left|\lambda_{2}\right|\left|\lambda_{3}\right|>\frac{\bar{y}^{2}}{1+\bar{y}^{2}}\left|\lambda_{2}\right|\left|\lambda_{3}\right|=\frac{\bar{y}^{2}}{1+\bar{y}^{2}}\left|\lambda_{2}\right|^{2},
$$

where $\left|\lambda_{2}\right|=\left|\lambda_{3}\right|$. Then $\left|\lambda_{2}\right|^{2}<1$ and so $\left|\lambda_{2}\right|=\left|\lambda_{3}\right|<1$ which completes the proof.

(2) The proof is similar to that of $1(b)$ and will be omitted.

\section{Periodicity and semicycle analysis of equation (1.6)}

Theorem 3.1. Let $\left\{y_{n}\right\}_{n=-2}^{\infty}$ be a nontrivial solution of equation (1.6) and let $\bar{y}$ denote the unique positive equilibrium of equation (1.6). Then the following statements are true:

(1) Assume that either,

$\left(C_{1}\right) y_{-1}<\bar{y} \leq y_{-2}, y_{0}$

or

$\left(C_{2}\right) y_{-2}, y_{0}<\bar{y} \leq y_{-1}$

is satisfied. Then $\left\{y_{n}\right\}_{n=-2}^{\infty}$ oscillates about $\bar{y}$ with semicycles of length one.

(2) Equation (1.6) has the periodic solution ..., $\varphi, \psi, \varphi, \psi, \ldots$ where $\varphi, \psi$ are the roots of the equation

$$
t^{2}+\frac{1}{\beta-1} t-\beta+1=0
$$

if $\beta<1$ and $\alpha>2(1-\beta)^{\frac{3}{2}}$.

\section{Proof.}

(1) Clear and will be omitted. 
(2) Let $\{\ldots, \varphi, \psi, \varphi, \psi, \ldots\}$ be a periodic solution of equation (1.6) with $\beta<1$. This implies that $\varphi=\frac{\alpha+\beta \varphi}{1+\psi^{2}}, \psi=\frac{\alpha+\beta \psi}{1+\varphi^{2}}$. Hence we have, $\varphi \psi=1-\beta$ and $\varphi+\psi=\frac{\alpha}{1-\beta}$. Therefore, $\varphi, \psi$ are the roots of the equation

$$
t^{2}+\frac{1}{\beta-1} t-\beta+1=0 .
$$

Now consider the discriminate $L=\left(\frac{\alpha}{1-\beta}\right)^{2}-4(1-\beta)$. It is clear that $L>0$ if $\beta<1$ and $\alpha>2(1-\beta)^{\frac{3}{2}}$.

\section{Global behavior of equation (1.6)}

This section is devoted to study the global stability of the positive equilibrium point $\bar{y}$ and the existence of unbounded solutions under certain conditions.

Theorem 4.1. Assume that $\beta<1$ and $\alpha<2(1-\beta)^{\frac{3}{2}}$. Then the positive equilibrium point $0<$ $\bar{y}<\sqrt{1-\beta}$ is globally asymptotically stable.

Proof. Let $\left\{y_{n}\right\}_{n=-2}^{\infty}$ be a solution of equation (1.6). Then

$$
y_{n+1}=\frac{\alpha+\beta y_{n-1}}{1+y_{n} y_{n-2}}<\alpha+\beta y_{n-1}, \quad n=0,1,2, \ldots
$$

It follows that there exists a real number $\gamma>0$ such that $y_{n}<\gamma, n \geq-2$.

This implies that

$$
y_{n+1}=\frac{\alpha+\beta y_{n-1}}{1+y_{n} y_{n-2}}>\frac{\alpha}{1+\gamma^{2}} .
$$

Let $\lambda=\liminf y_{n}$ and $\Lambda=\limsup y_{n}$. Hence we have

$$
\frac{\alpha+\beta \lambda}{1+\Lambda^{2}} \leq \lambda \leq \Lambda \leq \frac{\alpha+\beta \Lambda}{1+\lambda^{2}} .
$$

This implies that

$$
\alpha+\beta \lambda \leq \lambda+\lambda \Lambda^{2}
$$

and

$$
\Lambda+\Lambda \lambda^{2} \leq \alpha+\beta \Lambda
$$

Then

$$
\alpha \lambda+\beta \lambda^{2} \leq \lambda^{2}+\lambda^{2} \Lambda^{2}
$$

and

$$
\Lambda^{2}+\Lambda^{2} \lambda^{2} \leq \alpha \Lambda+\beta \Lambda^{2} .
$$

Hence we get that

$$
\alpha \lambda+\lambda^{2}(\beta-1) \leq \alpha \Lambda+\Lambda^{2}(\beta-1) .
$$


That is

$$
\lambda^{2}(1-\beta)-\alpha \lambda \geq \Lambda^{2}(1-\beta)-\alpha \Lambda .
$$

Consider the function $h(x)=(1-\beta) x^{2}-\alpha x$. As $\alpha<2(1-\beta)^{\frac{3}{2}}$, we have $\frac{\alpha}{2(1-\beta)}<\bar{y}<\sqrt{1-\beta}$, and $h(x)$ is increasing on $\left(\frac{\alpha}{2(1-\beta)}, \infty\right)$. In view of equation (4.1), we have a contradiction. Therefore $\lambda=\Lambda=\bar{y}$. This completes the proof.

Lemma 4.2. Assume that $\beta>2$ and $\alpha>0$. Then the following statements are true:

(1) If $x>\sqrt{\beta-1}+\frac{\alpha}{\sqrt{\beta-1}}$, then $\sqrt{\beta-1}>\frac{\alpha}{x^{2}-\beta+1}$.

(2) If $x>\sqrt{\beta-1}$ and $y>\frac{\alpha}{x^{2}-\beta+1}$, then $y>\frac{\alpha+\beta y}{1+x^{2}}$.

Theorem 4.3. Assume that $\beta>2$. Then equation (1.6) has solutions which are neither bounded nor persist.

Proof. Let $\left\{y_{n}\right\}_{n=-2}^{\infty}$ be a solution of equation (1.6) with initial conditions $\frac{\alpha}{y_{-1}^{2}-\beta+1}<y_{0}<y_{-2}<$ $\sqrt{\beta-1}$ and $y_{-1}>\sqrt{\beta-1}+\frac{\alpha}{\sqrt{\beta-1}}$. Then

$$
y_{1}=\frac{\alpha+\beta y_{-1}}{1+y_{0} y_{-2}}>\frac{\alpha+\beta y_{-1}}{\beta}=\frac{\alpha}{\beta}+y_{-1},
$$

where $y_{0} y_{-2}<\beta-1$.

$$
y_{2}=\frac{\alpha+\beta y_{0}}{1+y_{1} y_{-1}}<\frac{\alpha+\beta y_{0}}{1+y_{-1}^{2}}<y_{0},
$$

where $y_{1}>y_{-1}, y_{-1}>\sqrt{\beta-1}+\frac{\alpha}{\sqrt{\beta-1}}$, and $y_{0}>\frac{\alpha}{y_{-1}^{2}-\beta+1}$ (using lemma (4.2) (2)).

Now suppose that

$$
\frac{\alpha}{y_{2 n-1}^{2}-\beta+1}<y_{2 n}<y_{2 n-2}<\sqrt{\beta-1}
$$

and

$$
y_{2 n-1}>\sqrt{\beta-1}+\frac{\alpha}{\sqrt{\beta-1}} .
$$

Then

$$
y_{2 n+1}=\frac{\alpha+\beta y_{2 n-1}}{1+y_{2 n} y_{2 n-2}}>\frac{\alpha+\beta y_{2 n-1}}{\beta}=\frac{\alpha}{\beta}+y_{2 n-1}
$$

where $y_{2 n} y_{2 n-2}<y_{2 n-2}^{2}<\beta-1$, and

$$
y_{2 n+2}=\frac{\alpha+\beta y_{2 n}}{1+y_{2 n+1} y_{2 n-1}}<\frac{\alpha+\beta y_{2 n}}{1+y_{2 n-1}^{2}}<y_{2 n},
$$

where $y_{2 n+1}>y_{2 n-1}, y_{2 n-1}>\sqrt{\beta-1}+\frac{\alpha}{\sqrt{\beta-1}}$, and $y_{2 n}>\frac{\alpha}{y_{2 n-1}^{2}-\beta+1}$. Finally from equation (4.2) we have

$$
y_{2 n+1}>\frac{\alpha}{\beta}+y_{2 n-1}>\frac{\alpha}{\beta}+\left(\frac{\alpha}{\beta}+y_{2 n-3}\right)>\cdots>(n+1) \frac{\alpha}{\beta}+y_{-1} .
$$


If $\lim _{n \rightarrow \infty} y_{2 n+1}=L$ and $\lim _{n \rightarrow \infty} y_{2 n+2}=M$, then $L=\lim _{n \rightarrow \infty} y_{2 n+1}=\infty$ and $M=\lim _{n \rightarrow \infty}$ $y_{2 n+2}=\lim _{n \rightarrow \infty} \frac{\alpha+\beta y_{2 n}}{1+y_{2 n+1} y_{2 n-1}}=0$. This completes the proof.

5. Case $\beta=0$

When $\beta=0$, equation (1.6) becomes

$$
y_{n+1}=\frac{\alpha}{1+y_{n} y_{n-2}}, \quad n=0,1,2, \ldots
$$

It is clear that equation (5.1) has the unique positive equilibrium point $0<\bar{y}<\alpha$.

Theorem 5.1. (1) If $\alpha<2$, then the equilibrium point $\bar{y}$ is locally asymptotically stable.

(2) If $\alpha>2$, then the equilibrium point $\bar{y}$ is unstable (saddle point).

Proof. It is sufficient to consider the linearized equation

$$
z_{n+1}+\frac{\bar{y}^{2}}{1+\bar{y}^{2}}\left(z_{n}+z_{n-2}\right)=0, \quad n=0,1,2, \ldots
$$

and its associated characteristic equation

$$
\lambda^{3}+\frac{\bar{y}^{2}}{1+\bar{y}^{2}}\left(\lambda^{2}+1\right)=0
$$

Theorem 5.2. The following statements are true:

(1) Every solution of equation (5.1) is bounded and persists.

(2) Assume that $\alpha<2$. Then the equilibrium point $\bar{y}$ is globally asymptotically stable.

(3) Assume that $\alpha>2$. Then equation (5.1) has the unique periodic solution $\{\ldots, \varphi, \psi, \varphi, \psi, \ldots\}$, where $\varphi, \psi$ are the roots of the equation

$$
t^{2}-\alpha t+1=0
$$

\section{Proof.}

(1) It is clear that $\frac{\alpha}{1+\alpha^{2}} \leq y_{n+1} \leq \alpha, n=0,1,2, \ldots$

The proof of (2) and (3) are easy to establish and will be omitted.

\section{References}

[1] R. P. Agarwal, Difference Equations and Inequalities, First Edition, Marcel Dekker, 1992.

[2] M. A. Al-Shabi, R. Abo-Zeid, Global asymptotic stability of a higher order difference equation, Appl. Math. Sci., 4(17)(2010), 839-847.

[3] C. Cinar, on the positive solution of the difference equation $x_{n+1}=\frac{a x_{n-1}}{1+b x_{n} x_{n-1}}$, Appl. Math. Comput., 150(2004), 21-24. 
[4] E. A. Grove and G. Ladas, Periodicities in Nonlinear Difference Equations, Chapman and Hall/CRC, 2005.

[5] E. A. Grove, G. Ladas, M. Predescu and M. Radin, On the global character of the difference equation $x_{n+1}=$ $\frac{\alpha+\gamma x_{n-2 k+1}+\delta x_{n-2 l}}{A+x_{n-2 l}}$, J. Diff. Eq. App., 9(2)(2003), 171-199.

[6] C. H. Gibbons, M. R. S. Kulenović and G. Ladas, On the recursive sequence $x_{n+1}=\frac{\alpha+\beta x_{n-1}}{\gamma+x_{n}}$, Math. Sci. Res. Hot-Line, 4(2) (2000), 1-11.

[7] V. L. Kocic and G. Ladas, Global Behavior of Nonlinear Difference Equations of Higher Order with applications, Kluwer Academic, Dordrecht, 1993.

[8] V. L. Kocic and G. Ladas, Global attractivity in a second order nonlinear difference equations, J. Math. Anal. Appl., 180(1993), 144-150.

[9] M. R. S. Kulenovic, G. Ladas and N. P. Prokup, A rational difference equation, Comp. Math. Appl., 41(2001), 671-678.

[10] M. R. S. Kulenović and G. Ladas, Dynamics of Second-Order Rational Difference Equations; With Open Problems and Conjectures, Chapman and Hall/HRC Boca Raton, 2002.

[11] E. Camouzis and G. Ladas, Dynamics of Third-Order Rational Difference Equations; With Open Problems and Conjectures, Chapman and Hall/HRC Boca Raton, 2008.

[12] H. Sedaghat, Nonlinear Difference Equations, Theory and Applications to Social Science Models, Kluwer Academic Publishers, Dordrecht, 2003.

[13] X. Yang, W. Su, B. Chen, G.M. Megson, D.J. Evans, On the recursive sequence $x_{n+1}=\frac{a x_{n}+b x_{n-1}}{c+d x_{n} x_{n-1}}$, Appl. Math. Comput., 162(2005), 1485-1497.

Department of Basic Science, faculty of Engineering, October 6 university, 6th of October Governorate, Egypt.

E-mail: abuzead73@yahoo.com

Department of Computer Science, College of Computer, Qassim University, Buraidah, 51411, Saudi Arabia.

E-mail: malshabi@yahoo.com 